\title{
TIME COURSE OF REVERSE REMODELING OF THE LEFT VENTRICLE DURING SUPPORT WITH A LEFT VENTRICULAR ASSIST DEVICE
}

John D. Madigan, BA ${ }^{b}$

Alessandro Barbone, $\mathrm{MD}^{\mathrm{b}}$

Asim F. Choudhri, BS ${ }^{\mathrm{b}}$

David L. S. Morales, MD

Bolin Cai, MD

Mehmet C. Oz, MD

Daniel Burkhoff, MD, $\mathrm{PhD}^{\mathrm{a}}$
Background: Support with a left ventricular assist device leads to normalization of left ventricular chamber geometry, regression of myocyte hypertrophy, alterations in left ventricular collagen content, and normalized expression of genes involved with excitation-contraction coupling in patients with heart failure. The objective of this study was to investigate the time course of these processes.

Methods: Passive left ventricular pressure-volume relationships were obtained from explanted hearts of 19 patients with heart failure undergoing transplantation without left ventricular assist device support, 25 patients with heart failure supported before transplantation (duration of support ranging between 8 and 155 days), and 5 normal human hearts not suitable for transplantation. Left ventricular size was indexed by the volume at which left ventricular pressure reached $30 \mathrm{~mm} \mathrm{Hg}$. Left ventricular tissue samples were probed for sarcoplasmic endoreticular calcium adenosine triphosphatase $2 \mathrm{a}$ expression and processed for analysis of myocyte diameter and relative myocardial collagen content.

Results: The volume at which left ventricular pressure reached $30 \mathrm{~mm} \mathrm{Hg}$ was not significantly different between hearts without and with assist device support for less than 40 days. However, the volume at which left ventricular pressure reached $30 \mathrm{~mm} \mathrm{Hg}$ in patients with assist devices supported for more than 40 days was significantly smaller than that of the hearts without assist devices but was larger than that of normal hearts. A similar pattern was observed for myocyte diameter. Sarcoplasmic endoreticular calcium adenosine triphosphatase 2a expression increased to normal levels by about 20 days of support with an assist device. Relative collagen content was significantly increased in hearts supported for more than 40 days.

Conclusion: Maximum structural reverse remodeling by left ventricular assist devices is complete by about 40 days. Molecular reverse remodeling of sarcoplasmic endoreticular calcium adenosine triphosphatase $2 \mathrm{a}$ expression is quicker, being complete by about 20 days. (J Thorac Cardiovasc Surg 2001;121:902-8)
$\mathrm{L}_{\mathrm{b}}^{\mathrm{e}}$ eft ventricular assist devices (LVADs) are used as a bridge to transplantation for patients with severe decompensated heart failure. It has been demonstrated that the pressure and volume unloading of the left ventricle provided by LVADs can lead to normalization of left ventricular (LV) geometry, ${ }^{1}$ regression of $\mathrm{LV}$

From the Departments of Medicine ${ }^{\mathrm{a}}$ and Surgery, ${ }^{\mathrm{b}}$ Columbia University, New York, NY.

Copyright () 2001 by The American Association for Thoracic Surgery

$0022-5223 / 2001 \$ 35.00+0 \quad \mathbf{1 2 / 1 / 1 1 2 6 3 2}$

doi: $10.1067 / \mathrm{mtc} .2001 .112632$ myocyte hypertrophy, ${ }^{2}$ alterations in LV myocardial collagen content, and normalized expression of genes involved with excitation-contraction coupling. ${ }^{3}$ Collectively, these changes have been referred to as reverse remodeling ${ }^{1}$ and, in some circumstances, lead to improved intrinsic myocyte strength. ${ }^{3,4}$ In a small number of cases, the reverse remodeling leads to recovery of chamber strength of sufficient magnitude to permit LVAD explantation without the need for transplantation. ${ }^{5-7}$ Although clinical outcomes after LVAD explant have not been uniformly good ${ }^{6}$ and LVAD explant is not routine in the United States, understanding the process of reverse remodeling is important. Such understanding 
Table I. Patient demographics

\begin{tabular}{|c|c|c|c|c|}
\hline Parameter & Non-LVAD & $\operatorname{LVAD}(\text { all })^{*}$ & $L V A D_{0-40} *$ & $L V A D_{40+} *$ \\
\hline No. & 19 & 25 & 6 & 19 \\
\hline Cause (ICM/DCM/other) & $9 / 10 / 0$ & $8 / 14 / 3$ & $2 / 4 / 0$ & $6 / 10 / 3$ \\
\hline Age (y) & $51.5 \pm 12.9$ & $52 \pm 13$ & $53.1 \pm 5.5$ & $51.5 \pm 14.3$ \\
\hline $\operatorname{BSA}\left(\mathrm{m}^{2}\right)$ & $1.9 \pm 0.2$ & $1.9 \pm 0.2$ & $2.1 \pm 0.3$ & $1.8 \pm 0.1$ \\
\hline $\mathrm{EF}(\%)$ & $18 \pm 4$ & $18 \pm 5$ & $16 \pm 7$ & $18 \pm 4$ \\
\hline LV EDD (cm) & $6.9 \pm 0.6$ & $7.2 \pm 1.3$ & $7.2 \pm 1.3$ & $6.9 \pm 0.6$ \\
\hline PCWP (mm Hg) & $25 \pm 10$ & $27 \pm 8$ & $25 \pm 13$ & $25 \pm 10$ \\
\hline Duration of LVAD support & NA & $88 \pm 44$ & $21 \pm 11$ & $100 \pm 33$ \\
\hline
\end{tabular}

$I C M$, Ischemic cardiomyopathy; DCM, dilated idiopathic cardiomyopathy; $B S A$, body surface area; $E F$, ejection fraction; EDD, end-diastolic diameter; $P C W P$, pulmonary capillary wedge pressure; $N A$, not applicable.

*All values for patients with LVADs are those at the time of LVAD implantation, whereas values for the non-LVAD patients are those at the time of transplantation.

will lead to new insights into fundamental mechanisms underlying heart failure, the extent to which pathophysiologic processes can be reversed, and the potential role of LVAD therapy as an adjunct to other therapies aimed at curing heart failure. More recent evidence suggesting that long-term outcome after LVAD explantation could be improved with appropriate patient selection and weaning protocols ${ }^{7}$ make improved understanding of the process even more important.

Current understanding of LVAD-induced reverse remodeling is rudimentary. One fundamental unexplored aspect of this multifaceted process is the time course with which it occurs. The objective of this study was therefore to define the time course of structural, cellular, interstitial, and molecular reverse remodeling of the left ventricle during LVAD support.

\section{Methods}

Heart harvest and pressure-volume relationships. Under a protocol approved by the Institutional Review Board of the Columbia Presbyterian Medical Center, 49 human hearts were used for this study. Hearts from 19 patients with end-stage congestive heart failure (CHF) and 25 patients with end-stage CHF supported by a Thermo Cardiosystems, Inc (TCI, Woburn, Mass) LVAD for varying amounts of time were obtained at the time of cardiac transplantation. Five normal donor hearts unsuitable for transplantation were also obtained.

All hearts were perfused with cold, hypocalcemic, hyperkalemic cardioplegic solution at explantation. The passive LV pressure-volume relationship of each arrested heart was measured as described previously. ${ }^{3}$ In brief, the aortic root and, in the case of the LVAD-supported hearts, the LVAD inflow cannula were clamp occluded. A metal adapter was attached to the mitral anulus, and a compliant water-filled latex balloon was placed within the LV chamber. Pressure within the balloon was measured with a high-fidelity micromanometer as volume was progressively increased. The pressure was then plotted as a function of volume at each step, resulting in a passive LV pressure-volume relationship that is similar to the end-diastolic pressure-volume relationship of a contracting heart. Chamber size was indexed by the volume at which pressure within the ventricle reached $30 \mathrm{~mm} \mathrm{Hg}$ (LVV30).

Myocyte diameter and myocardial collagen content. Tissue samples were obtained from the LV free wall, fixed in $10 \%$ buffered formalin, embedded in paraffin, and mounted on glass slides. Samples were then prepared with Masson trichrome stain. Images were viewed on a Nikon microscope (Nikon Corporation, Tokyo, Japan) with an MTI 3CCD digital camera (Dage-MTI, Inc, Michigan City, Ind) at $20 \times$ magnification to assess myocyte diameter and at $4 \times$ magnification to assess myocardial collagen content. Digitally acquired images were analyzed with Image Pro Plus V3.0 software (Media Cybernetics, LP, Silver Spring, Md) by an examiner blinded to whether the heart was normal, failing, or LVAD supported. Two orthogonal diameters were obtained per myocyte and then averaged. Only sections containing fibers cut in cross-section were analyzed. The diameters of 50 myocytes per patient were measured and then averaged. Myocardial collagen content was assessed by using a green software filter. On each section, a density range was set to resemble the collagen content. The number of pixels included in this range was divided by the total number of pixels on the fields. Ten fields at $4 \times$ magnification per patient were analyzed, and results were then averaged.

Northern blot analysis. Tissue samples were obtained from the LV free wall and snap-frozen in liquid nitrogen for RNA extraction. Total RNA was extracted from the myocardial tissue samples with guanidinium thiocyanate followed by centrifugation in cesium chloride solutions. Aliquots $(25$ $\mu \mathrm{g}$ ) of total RNA quantified by absorbance at $260 \mathrm{~nm}$ were then separated by using electrophoresis in $1 \%$ agarose/15\% formaldehyde gels in $1 \times 3$-( $N$-morpholino) propanesulfonic acid buffer (Fisher Scientific, Hampton, NJ). After intensive capillary transfer to Nirto Pure nitrocellulose membranes (MSI, Westboro, Mass) in 20× standard saline citrate (SSC; $1 \times \mathrm{SSC}$ equals $3 \mathrm{~mol} / \mathrm{L}$ sodium chloride and $0.15 \mathrm{~mol} / \mathrm{L}$ sodium citrate, $\mathrm{pH} 7.0$ ), the RNA was fixed by baking in a vacuum oven at $80^{\circ} \mathrm{C}$ for 1 hour and prehybridized at $42^{\circ} \mathrm{C}$ for more than 3 hours in a solution of $50 \%$ formamide, $4 \times$ 


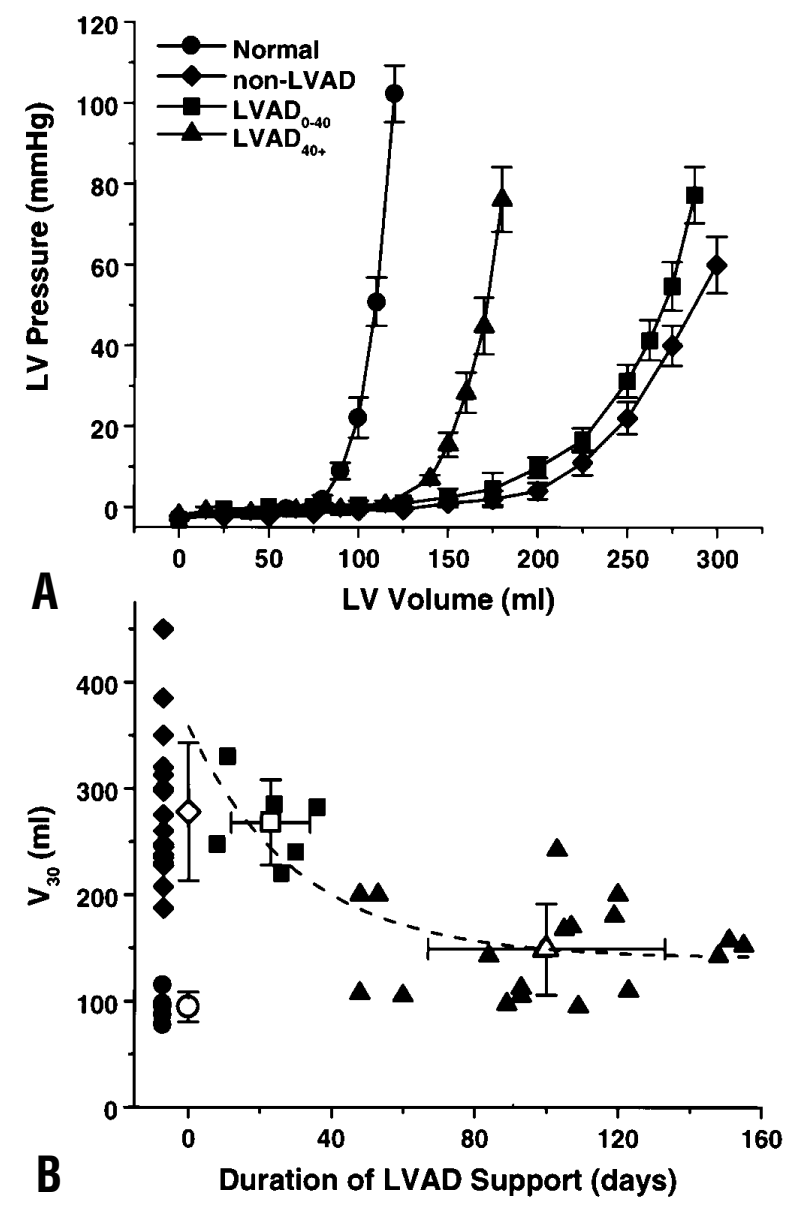

Fig 1. A, Passive pressure-volume relationships for the 4 groups (squares, $\mathrm{LVAD}_{0-40}$; triangles, $\mathrm{LVAD}_{40+}$; diamonds, non-LVAD; circles, normal). B, LVV30 versus duration of LVAD support (squares, $\mathrm{LVAD}_{0-40} ;$ triangles, $\mathrm{LVAD}_{40+} ;$ diamonds, non-LVAD; circles, normal; dashed line, nonlinear fit to LVAD data). See Table II for regression parameters.

SSC, $1 \times$ Denhardt solution, $0.1 \%$ sodium dodecylsulfate (SDS), $1 \mathrm{mmol} / \mathrm{L}$ ethylenediaminetetraacetic acid, and 0.125 $\mathrm{mg} / \mathrm{mL}$ denatured salmon testis DNA. The blots were hybridized overnight with cDNA for rat sarcoplasmic endoreticular calcium adenosine triphosphatase 2 (SERCA2; 1.181-kb EcoRI fragment, a generous gift from Dr Gerd Hasenfuss) and human reduced glyceraldehydephosphate dehydrogenase (GAPDH; 1.3-kb PstI fragment). All cDNA probes were labeled with a phosphorus 32-labeled deoxycytidine triphosphate $(3000 \mathrm{Ci} / \mathrm{mmol}$, Amersham, Arlington Heights, Ill) to a specific activity of 1 $\times 10^{6} \mathrm{cpm} / \mu \mathrm{g}$ by using a multiprimer DNA-labeling system (Amersham). The blots were washed twice in $2 \times$ SSC at room temperature for 15 minutes and 3 times in $0.1 \times$ $\mathrm{SSC} / 0.1 \% \mathrm{SDS}$ at $68^{\circ} \mathrm{C}$ for 15 minutes and exposed to $\mathrm{X}$ OMAT AR film (Eastman Kodak Company, Rochester, NY) at $-70^{\circ} \mathrm{C}$ with an intensifying screen for 48 to 72 hours. Autoradiograms were then analyzed by means of laser den- sitometry (Molecular Dynamics Inc, Sunnyvale, Calif) in the linear response range of the $\mathrm{x}$-ray films by using GAPDH as an internal standard. The relative SERCA2a expression was quantified as the ratio of SERCA2a band intensity to GAPDH band intensity. To be able to pool results from different gels, all values were further standardized to the average intensities of the same 3 normal samples that were included on every blot. Of the major proteins involved in calcium cycling that we have examined previously (which have included the ryanodine receptor and sodium-calcium exchanger in addition to SERCA2a), it is our experience that SERCA2a mRNA levels are the highest under normal conditions and thus provide the greatest reliability for detecting changes in expression. ${ }^{3}$

Data analysis. Data related to each parameter examined were graphed as a function of duration of LVAD support and fit to an exponential function as follows:

$$
\mathrm{y}=\mathrm{y}_{\mathrm{o}}+\alpha\left(1-\mathrm{e}^{-\mathrm{t} / \tau}\right)
$$

where $y$ is the parameter of interest, $y_{0}$ is the extrapolated value to 0 days of support, $t$ is the duration of LVAD support, $\alpha$ is the maximum magnitude of the change, and $\tau$ is the time constant for change. Fitting was done with a nonlinear Levenberg-Marquard algorithm. To determine whether these relationships where statistically significant, they were linearized by means of a standard logarithmic transformation. Linear regression analysis was then applied, which provided a $P$ value for the relationship.

As detailed further in the "Results" section, patients were divided into 4 groups on the basis of duration of support for the purpose of performing statistical comparisons. The normal group $(n=5)$ comprised hearts from normal donors. The non-LVAD group $(n=19)$ comprised hearts from patients in end-stage CHF who did not receive LVAD support before transplantation. The $\mathrm{LVAD}_{0-40}$ group $(\mathrm{n}=6)$ comprised hearts from patients with end-stage CHF supported for less than 40 days before transplantation. The $\mathrm{LVAD}_{40+}$ group $(\mathrm{n}=19)$ comprised hearts from patients with end-stage CHF supported for more than 40 days before transplantation. The choice of 40 days as the cutoff point for this grouping was based on the longest time constant of change determined from the nonlinear analysis. Differences between the groups were analyzed by means of 1 -factor analysis of variance with a TukeyKramer post hoc multiple comparisons test. All values are presented as means and SDs.

\section{Results}

Patient population. Patient demographics are summarized in Table I. The cause of CHF was similar in the non-LVAD and LVAD groups, with slightly more patients having dilated idiopathic cardiomyopathy than ischemic cardiomyopathy. All patients with LVADs were receiving inotropic and vasopressor therapies before LVAD insertion. This was not generally the case for patients in the non-LVAD group, some of whom were living at home before transplantation. 

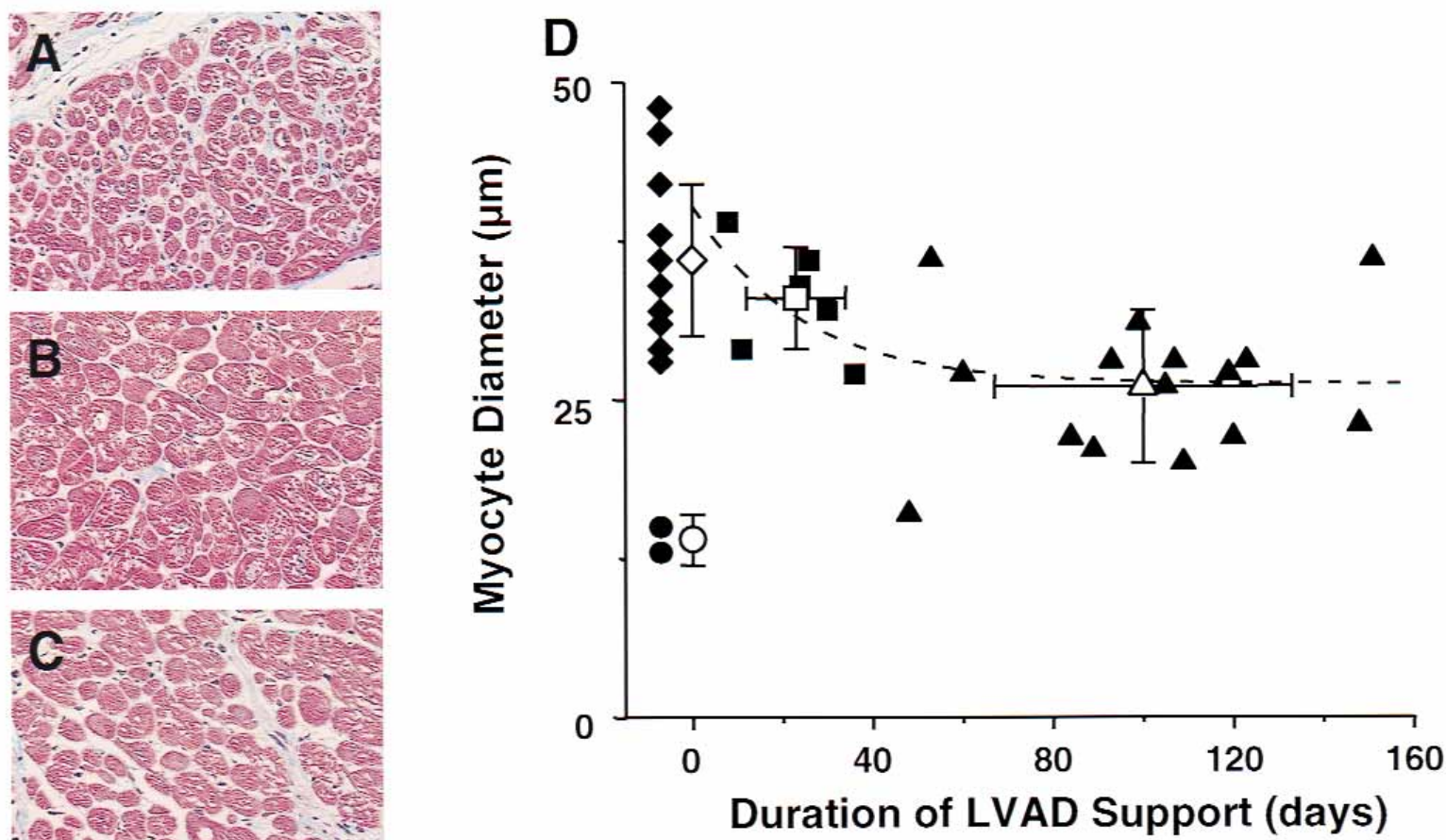

Table II. Parameter values for nonlinear time course of change in parameter values as a function of duration of LVAD support: $y=y_{o}+\alpha\left(1-e^{-t / \tau}\right)$

\begin{tabular}{lcccc}
\hline Parameter & LVV30 & Myocyte diameter & Collagen content & SERCA2 expression \\
\hline $\mathrm{y}_{\mathrm{o}}$ & 217.7 & 0.141 & -11.1 & -1.26 \\
$\alpha$ & 140.5 & 0.262 & 17.98 & 0.902 \\
$\tau$ & 30.8 & 23.7 & 39.2 & 15.1 \\
$P$ value & $<.0001$ & $<.0001$ & .0003 & .0421
\end{tabular}

$y_{o}$, Starting value was set at the mean value obtained from all non-LVAD transplanted hearts; $\alpha$, amplitude of the change; $\tau$, time constant of change. $P$ values are the statistical significance of the relationship between time and the specified variable.

None of the patients required mechanical biventricular support before LVAD insertion or transplantation, although several patients required support with intraaortic balloon counterpulsation. The mean duration of LVAD support was 82 days and ranged between 8 and 155 days. There were no statistically significant differences in preoperative body surface area, ejection fraction, left ventricular end-diastolic dimension, or pulmonary capillary wedge pressure between groups.

LVV30. The LVV30 value was plotted as a function of the duration of LVAD support, as shown in Fig 1, $B$. LVV30 decreased monotonically with increased duration of LVAD support, indicating progressive reverse structural remodeling of the chamber to a plateau value. LVV30 values of the patients in the non-LVAD group were similar to those in the LVAD group supported for short durations and were each significantly larger than LVV30 values of normal hearts. The nonlinear fit to the LVAD data (parameter values summarized in Table II) revealed a time constant of approximately 30 days. Statistical comparisons revealed no difference between LVV30 values of the non-LVAD and $\mathrm{LVAD}_{0-40}$ groups $(P=.14)$ and statistically significant differences between the non-LVAD and $\mathrm{LVAD}_{40+}$ groups $(P<.001)$ and $\mathrm{LVAD}_{0-40}$ and $\mathrm{LVAD}_{40+}$ 

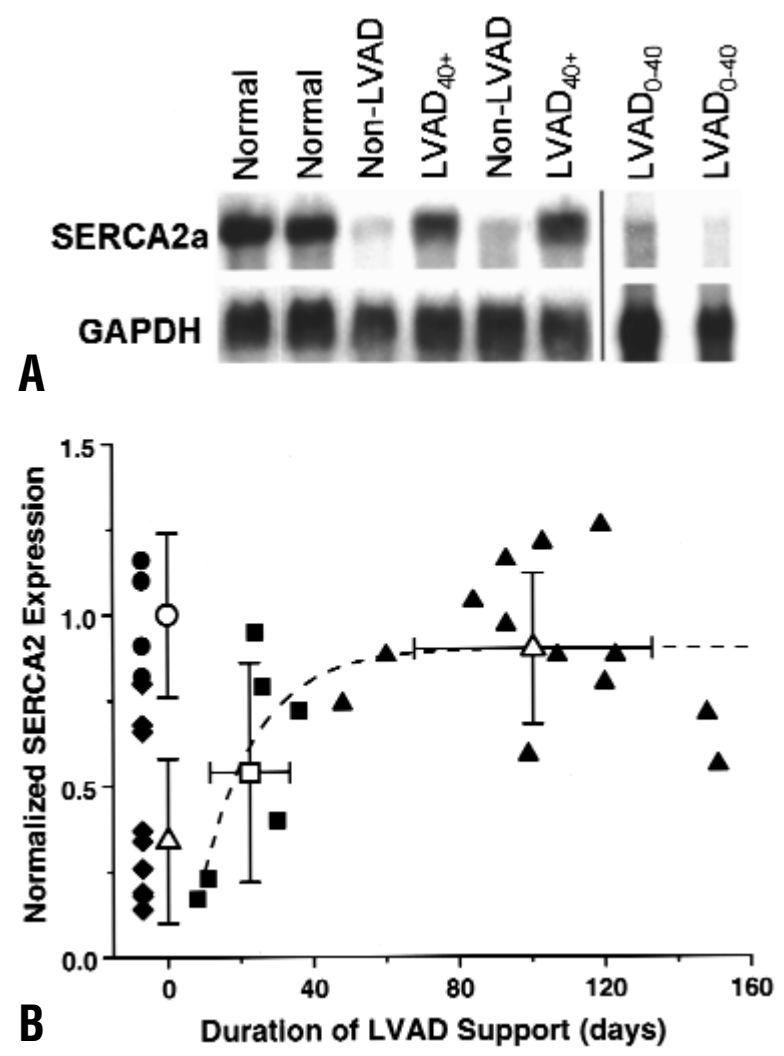

Fig 3. A, Typical northern blot demonstrating expression of SERCA2a relative to the GAPDH reference gene. B, Expression of SERCA2a versus duration of LVAD support (squares, $\mathrm{LVAD}_{0-40}$; triangles, $\mathrm{LVAD}_{40+}$; diamonds, nonLVAD; circles, normal; dashed line, nonlinear fit to LVAD data). See Table II for regression parameters.

groups $(P<.001)$ and between the control group and each of the other 3 groups $(P=.015)$. Thus although substantial chamber reverse remodeling had occurred with LVAD durations greater than 40 days, LVV30 on average was still greater than that of the normal hearts. These differences are shown more clearly in the mean passive pressure-volume curves for the normal, non-LVAD, $\operatorname{LVAD}_{0-40}$, and $\mathrm{LVAD}_{40+}$ hearts shown in Fig 1, $A$.

Myocyte diameter. The relationship between LV myocyte diameter and duration of support is shown in Fig 2, $D$ (format similar to that of Fig 1, B). The time constant of change of myocyte diameter was approximately 24 days. There was a significant difference between the non-LVAD and normal groups $(P<.001)$, and no statistically significant difference between the non-LVAD and $\operatorname{LVAD}_{0-40}$ groups $(P=.18)$. The $\mathrm{LVAD}_{40+}$ group exhibited significantly smaller diameters when compared with the non-LVAD group $(P=.002)$, although the values were still significantly larger than those in the normal hearts $(P=.01)$. Examples of histologic samples are shown in Fig 2, $A$ (normal), $B\left(\operatorname{LVAD}_{0-40}\right)$, and $C\left(\mathrm{LVAD}_{40+}\right)$.

SERCA2a expression. Typical examples of Northern blots obtained from these hearts are shown in Fig 3, A. The relationship between LV myocardial expression of SERCA2a expression and duration of support is shown in Fig 3, $B$ (format similar to that of Fig 1, $B$ ). As in previous studies, SERCA2a expression was significantly decreased in the non-LVAD patients undergoing CHF and in the $\mathrm{LVAD}_{0-40}$ group compared with the normal group $(P<.001$ and $P=.005$, respectively), and there was no statistically significant difference between the non-LVAD and $\operatorname{LVAD}_{0-40}$ groups $(P=.18)$. The $\mathrm{LVAD}_{40+}$ group, however, exhibited values significantly higher than those of the non-LVAD group $(P<.001)$ and reached values that were not different than those of the normal hearts $(P=.30)$. The time constant of change of SERCA2a determined by the nonlinear curve fitting was approximately 15 days, which is shorter than those of the other parameters examined.

Myocardial collagen content. The relationship between relative myocardial collagen content (defined as the percentage of sample area occupied by blue-staining collagen fibers on Trichromestained slides) and duration of support is shown in Fig 4, $D$ (format similar to that of Fig $1, B$ ). In contrast to the other parameters studied, there was no statistically significant difference between the nonLVAD and normal groups $(P=.24)$. There was also no statistically significant difference between the non-LVAD and $\operatorname{LVAD}_{0-40}$ groups $(P=.10)$. However, the $\mathrm{LVAD}_{40+}$ group exhibited values significantly higher than those of the non-LVAD group $(P<.001)$. The blinded nature of this analysis and the attempt to avoid areas of massively fibrotic areas should be reiterated. The time constant of change of interstitial collagen was about 40 days, which is slightly longer than the other parameters. Subgroup analysis showed that this finding was independent of the cause of CHF (ischemic cardiomyopathy vs dilated idiopathic cardiomyopathy). Thus LVAD support may lead to an increase in relative myocardial collagen content. Examples of histologic sample are shown in Fig 4, $A$ (normal), $B\left(\operatorname{LVAD}_{0-40}\right)$, and $C\left(\operatorname{LVAD}_{40+}\right)$.

\section{Discussion}

The present study confirms prior reports showing that reverse remodeling, including decreased chamber size, decreased myocyte size, and increased expression of the gene encoding for SERCA2a (an important enzyme pump involved in calcium cycling and excita- 

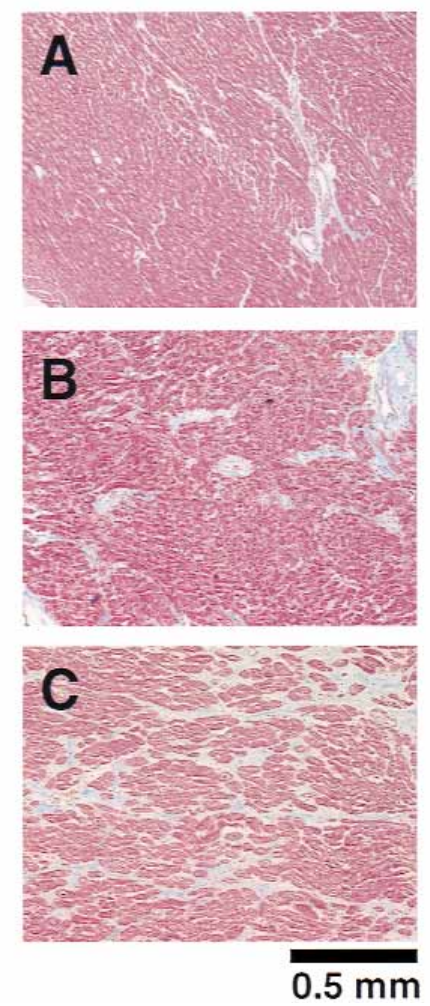

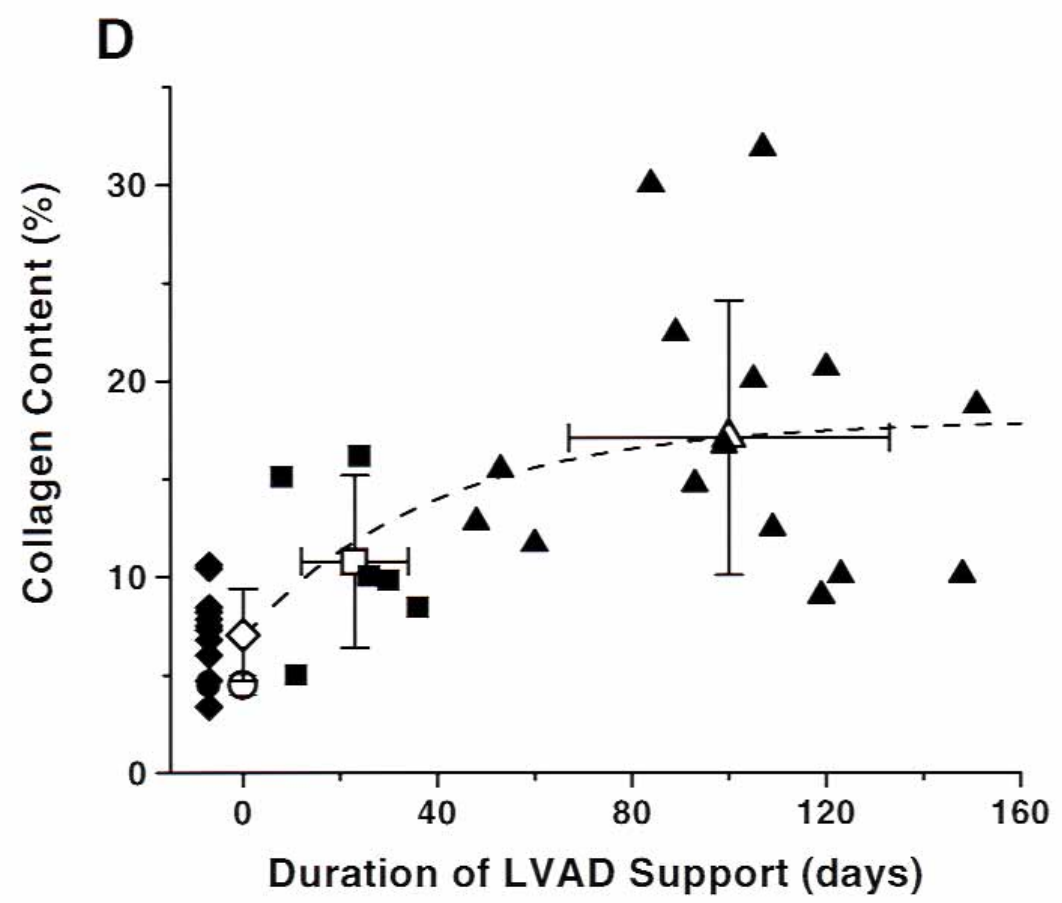

Fig 4. Trichrome-stained sections of LV free wall demonstrating relative myocardial collagen content for normal (A), non-LVAD (B), and $\mathrm{LVAD}_{40+}(\mathbf{C})$ groups. (Original magnification $4 \times$.) D, Relative myocardial collagen content versus duration of LVAD support (squares, $\mathrm{LVAD}_{0-40}$; triangles, $\mathrm{LVAD}_{40+}$; diamonds, non-LVAD; circles, normal; dashed line, nonlinear fit to LVAD data). See Table II for regression parameters. tion contraction coupling) occurs during LVAD support in a majority of patients, regardless of cause. The time constant of change for these parameters ranged between 15 and 30 days, and therefore the maximum benefit was observed by approximately 40 days. Structural aspects of reverse remodeling did not reach complete normalization, although SERCA2a expression did normalize.

Relative collagen content increased during LVAD support. It is likely that this is purely a result of the decrease in myocyte mass that occurs during LVAD support rather than increased deposition of collagen. It is clear that a decrease in myocyte mass with a fixed absolute amount of collagen would result in an increase by the same percentage of myocardium occupied by collagen. By using a simple model of myocardium composed only of myocytes and collagen, it can be shown quantitatively that a reduction of myocyte diameter from 35 to $25 \mu \mathrm{m}$ would be expected to increase relative collagen content from the measured starting value of $10 \%$ to a predicted value of $14 \%$ if the absolute amount of collagen remained constant. This is very close to the measured $15 \%$ collagen content determined for the $\mathrm{LVAD}_{40+}$ group by means of blinded analysis (Fig 4,D). Additionally, the half-life of collagen turnover is between 80 and 120 days, and therefore the patients with LVADs included in the present study did not have durations of support long enough to allow for significant alterations in collagen deposition or resorption. Significantly longer durations of support would be required to determine whether LVAD support influences absolute collagen content.

The precise mechanisms underlying the various aspects of reverse remodeling remain to be determined. The mechanisms underlying hypertrophy and chamber remodeling in response to pressure and volume loading of the myocardium involves intricately orchestrated upregulation and downregulation of a multitude of intracellular signaling cascades. ${ }^{8}$ Although investigated for over 20 years, these mechanisms are still not fully understood. It has been our working hypothesis that reverse remodeling involves the same mechanisms 
working in the opposite direction. In fact, it has been questioned whether reverse remodeling is simply a reflection of myocyte atrophy. The finding that, on average, myocyte diameters in the $\mathrm{LVAD}_{40+}$ group were still larger than normal and that the process of cellular size reduction appears to be completed by approximately 40 days despite continued off-loading of the muscle implies that there is a limit to how much atrophy the once diseased and hypertrophied myocyte can undergo.

Recent evidence suggests that in some patients, LVAD support may lead to improvement of global pump function of sufficient magnitude to permit explantation of the device without subsequent transplantation. ${ }^{5}$ This has led to the concept of using LVADs as a bridge to recovery. The potential of this possibility is strengthened by data demonstrating normalization of LV chamber geometry, ${ }^{1}$ regression of LV myocyte hypertrophy, ${ }^{2}$ increased myocyte contractile strength, enhanced inotropic response to adrenergic stimulation, and improved cytosolic calcium transients. ${ }^{4}$ However, in a recent article from our institution, there was a low incidence of myocardial recovery during LVAD support, as assessed by exercise testing with device output turned down, and the outcome of a small group of patients that underwent explantation was not uniformly good. ${ }^{6}$ Accordingly, weaning from LVAD support is not currently considered the standard of care, although more recent data suggest that outcome after LVAD explant may be improved. ${ }^{7}$ The goal of using LVADs as a bridge to recovery is a worthy pursuit because of the severe imbalance between the number of patients requiring transplant and the number of available donor hearts. Better understanding of the process of reverse remodeling will aid the development of adjunctive therapies, better patient selection criteria, and perhaps optimum LVAD use protocols to improve patient outcome after LVAD explantation. The data of the present study indicate that, as used currently, maximum benefits of LVADs in terms of reverse remodeling are observed after about 40 days, and little change is seen after this time point. In the short term a small increase in relative interstitial fibrosis may occur, not because of an increase in collagen deposition but because of the decrease in myocyte diameter with no significant change in total collagen content.

Received for publication March 15, 2000; revisions requested April 12, 2000; revisions received Oct 26, 2000; accepted for publication Oct 31, 2000.

Address for reprints: John D. Madigan, c/o Daniel Burkhoff, Columbia University, Department of Medicine, Black Building 812, 650 West 168th St, New York, NY 10032 (E-mail: jdm50@columbia.edu).

\section{REFERENCES}

1. Levin HR, Oz MC, Chen JM, Packer MP, Rose EA, Burkhoff D. Reversal of chronic ventricular dilation in patients with end-stage cardiomyopathy by prolonged mechanical unloading. Circulation 1995;91:2717-20.

2. Zafeiridis A, Jeevanandam V, Houser SR, Margulies KB. Regression of cellular hypertrophy after left ventricular assist device support. Circulation 1998;98:656-62.

3. Heerdt PM, Holmes JW, Cai B, Barbone A, Madigan JD, Reiken $\mathrm{S}$, et al. Chronic unloading by left ventricular assist device (LVAD) reverses contractile dysfunction and alters gene expression in end-stage heart failure. Circulation 2000;102:2713-9.

4. Dipla K, Mattiello JA, Jeevanandam V, Houser SR, Margulies $\mathrm{KB}$. Myocyte recovery after mechanical circulatory support in humans with end-stage heart failure. Circulation 1998;97:231622.

5. Muller J, Wallukat G, Weng YG, Dandel M, Spiegelsberger S, Semrau S, et al. Weaning from mechanical cardiac support in patients with idiopathic dilated cardiomyopathy. Circulation 1997;96:542-9.

6. Mancini DM, Beniaminvitz A, Levin HR, Catonese K, Flannery M, DiTullio M, et al. Low incidence of myocardial recovery after left ventricular assist device implantation in patients with chronic heart failure. Circulation 1998;98:2383-9.

7. Hetzer R, Mueller J, Weng Y, Wallukat G, Spiegelsberger S, Loebe M. Cardiac recovery in dilated cardiomyopathy by unloading with a left ventricular assist device. Ann Thorac Surg 1999;68:742-9.

8. Sodoshima J, Izumo S. The cellular and molecular response of cardiac myocytes to mechanical stress. Annu Rev Physiol 1997;59:551-71. 